MZ-TH/97-18

\title{
Chiral anomaly in contorted spacetimes
}

\author{
Eckehard W. Mielke ${ }^{\diamond *}$ and Dirk Kreimer ${ }^{\$ \dagger}$ \\ $\diamond$ Departamento de Física, \\ Universidad Autónoma Metropolitana-Iztapalapa, \\ Apartado Postal 55-534, C.P. 09340, México, D.F., MEXICO \\ \$ Institut für Physik, \\ Universität Mainz \\ 55099 Mainz, GERMANY
}

\begin{abstract}
The Dirac equation in Riemann-Cartan spacetimes with torsion is reconsidered. As is well-known, only the axial covector torsion $A$, a one-form, couples to massive Dirac fields. Using diagrammatic techniques, we show that besides the familiar Riemannian term only the Pontrjagin type four-form $d A \wedge d A$ does arise additionally in the chiral anomaly, but not the Nieh-Yan term $d^{*} A$, as has been claimed recently. Implications for cosmic strings in Einstein-Cartan theory as well as for Ashtekar's canonical approach to quantum gravity are discussed.
\end{abstract}

PACS no.: 04.50.+h; 04.20.Jb; 03.50.Kk

*E-mail: ekke@xanum.uam.mx

${ }^{\dagger}$ E-mail: kreimer@dipmza.physik.uni-mainz.de 


\section{INTRODUCTION}

Quantum anomalies both in the Riemannian and in the Riemann-Cartan spacetimes were calculated previously in several papers using different methods, see e.g. [5, 11, 40, 41,7]. However, recently Chandia and Zanelli [6] have questioned the completeness of the earlier calculations which all demonstrated that the Nieh-Yan four-form [33] is irrelevant to the axial anomaly.

For the axial anomaly, we have a couple of distinguished features. Most prominent is its relation with the Atiyah-Singer index theorem [3]. But also from the viewpoint of perturbative quantum field theory, the chiral anomaly has some features which signal its conceptual importance. There is the remarkable fact that it does not renormalize - higher order loop corrections do not alter its one-loop value. This very fact guarantees that the anomaly can be given a topological interpretation. Another feature is its finiteness: in any approach, the chiral anomaly as a topological invariant is a finite quantity.

Chandia and Zanelli argue that the Nieh-Yan four-form will add to this quantity. As usual, they confront the fact that such a term, if it is generated at all, is ill-defined, by whatever regulator one uses. In their case, they use a Fujikawa-type approach and propose to absorb the regulator mass in a rescaled vierbein. We will present arguments which question the validity of such an approach.

\section{GRAVITATIONAL CHERN-SIMONS AND PONTRJAGIN TERMS}

We are using Clifford-algebra valued exterior forms [28,31], in which the constant Dirac matrices $\gamma_{\alpha}$ obeying $\gamma_{\alpha} \gamma_{\beta}+\gamma_{\beta} \gamma_{\alpha}=2 o_{\alpha \beta}$ are saturating the index of the orthonomal coframe one-form $\vartheta^{\alpha}$ and its Hodge dual $\eta^{\alpha}:={ }^{*} \vartheta^{\alpha}$ via:

$$
\gamma:=\gamma_{\alpha} \vartheta^{\alpha}, \quad{ }^{*} \gamma=\gamma^{\alpha} \eta_{\alpha}
$$

In terms of the Clifford algebra-valued connection $\Gamma:=\frac{i}{4} \Gamma^{\alpha \beta} \sigma_{\alpha \beta}$, the $S L(2, C)$-covariant exterior derivative is given by $D=d+\Gamma \wedge$, where $\sigma_{\alpha \beta}=\frac{i}{2}\left(\gamma_{\alpha} \gamma_{\beta}-\gamma_{\beta} \gamma_{\alpha}\right)$ are the Lorentz 
generators entering also in the Clifford-algebra valued two-form $\sigma:=\frac{i}{2} \gamma \wedge \gamma=\frac{1}{2} \sigma_{\alpha \beta} \vartheta^{\alpha} \wedge \vartheta^{\beta}$.

Differentiation of these basic variables leads to the Clifford algebra-valued torsion and curvature two-forms:

$$
\Theta:=D \gamma=T^{\alpha} \gamma_{\alpha}, \quad \Omega:=d \Gamma+\Gamma \wedge \Gamma=\frac{i}{4} R^{\alpha \beta} \sigma_{\alpha \beta}
$$

In Riemann-Cartan (RC) geometry, the Chern-Simons term for the Lorentz connection reads 114

$$
C_{\mathrm{RR}}:=-\operatorname{Tr}\left(\Gamma \wedge \Omega-\frac{1}{3} \Gamma \wedge \Gamma \wedge \Gamma\right)
$$

The corresponding Pontrjagin term can be obtained by exterior differentiation

$$
d C_{\mathrm{RR}}=-\operatorname{Tr}(\Omega \wedge \Omega)=\frac{1}{2} R^{\alpha \beta} \wedge R_{\alpha \beta}
$$

Since the coframe can be regarded as the translational part of the Cartan connection [30,15,19, a related translational Chern-Simons term arises

$$
C_{\mathrm{TT}}:=\frac{1}{8 \ell^{2}} \operatorname{Tr}(\gamma \wedge \Theta)=\frac{1}{2 \ell^{2}} \vartheta^{\alpha} \wedge T_{\alpha}
$$

By exterior differentiation we obtain the Nieh-Yan four-form [33]:

$$
d C_{\mathrm{TT}}=\frac{2}{\ell^{2}}\left(T^{\alpha} \wedge T_{\alpha}+R_{\alpha \beta} \wedge \vartheta^{\alpha} \wedge \vartheta^{\beta}\right)
$$

A fundamental length $\ell$ introduced here is necessary for dimensional reasons. This is also motivated by a de Sitter type [10] approach, in which the $\operatorname{sl}(5, R)$-valued connection $\hat{\Gamma}=\Gamma+(1 / \ell)\left(\vartheta^{\alpha} L^{4}{ }_{\alpha}+\vartheta_{\beta} L^{\beta}{ }_{4}\right)$ is expanded into the dimensionless linear connection $\Gamma$ plus the coframe with canonical dimension [length]. The corresponding Pontrjagin term $\hat{C}_{\mathrm{RR}}$ splits via

$$
\hat{C}_{\mathrm{RR}}=C_{\mathrm{RR}}-2 C_{\mathrm{TT}}
$$

into the linear one and the translational Chern-Simons term, see the footnote 31 of Ref. [15]. (This relation has recently been "recovered" by Chandia et al. [6]). 


\section{DIRAC FIELDS IN RIEMANN-CARTAN SPACETIME}

The Dirac Lagrangian is given by the manifestly Hermitian four-form

$$
L_{\mathrm{D}}=L(\gamma, \psi, D \psi)=\frac{i}{2}\left\{\bar{\psi}^{*} \gamma \wedge D \psi+\overline{D \psi} \wedge^{*} \gamma \psi\right\}+{ }^{*} m \bar{\psi} \psi,
$$

for which $\bar{\psi}:=\psi^{\dagger} \gamma_{0}$ is the Dirac adjoint and ${ }^{*} m=m \eta$ the mass term, cf. [28].

The Dirac equation and its adjoint are obtained by varying $L_{\mathrm{D}}$ independently with respect to $\bar{\psi}$ and $\psi$ :

$$
\begin{aligned}
i^{*} \gamma \wedge D \psi+{ }^{*} m \psi-\frac{i}{2}\left(D^{*} \gamma\right) \psi & =0 \\
i \overline{D \psi} \wedge{ }^{*} \gamma+{ }^{*} m \bar{\psi}+\frac{i}{2} \bar{\psi} D^{*} \gamma & =0
\end{aligned}
$$

If we make use of the properties of the Hodge dual and the torsion $\Theta:=D \gamma$, the Dirac equation assumes the equivalent form

$$
i^{*} \gamma \wedge\left(D+\frac{i}{4} m \gamma-\frac{1}{2} T\right) \psi=0
$$

where

$$
\left.\left.\left.T:=\frac{1}{4} \operatorname{Tr}(\check{\gamma}\rfloor \Theta\right)=e_{\alpha}\right\rfloor T^{\alpha}, \quad A:=\frac{1}{4} \operatorname{Tr}(\check{\gamma}\rfloor^{*} \Theta\right)=\frac{1}{4}{ }^{*} \operatorname{Tr}(\gamma \wedge \Theta)={ }^{*}\left(\vartheta^{\alpha} \wedge T_{\alpha}\right) .
$$

are the one-forms of the trace and axial vector torsion, respectively.

Note that torsion is also hidden in the RC covariant derivative $D$. In order to separate out the purely Riemannian piece from torsion terms, we decompose the Riemann-Cartan connection $\Gamma=\Gamma^{\{\}}-K$ into the Riemannian (or Christoffel) connection $\Gamma^{\{\}}$and the contortion one-form $K=\frac{i}{4} K^{\alpha \beta} \sigma_{\alpha \beta}$, obeying $\Theta=-[K, \gamma]$. Accordingly, the Dirac Lagrangian (3.1) splits into a Riemannian and a spin-contortion piece, cf. Refs. [28,31:

$$
\begin{aligned}
L_{\mathrm{D}} & =L\left(\gamma, \psi, D^{\{\}} \psi\right)-\frac{i}{2} \bar{\psi}\left({ }^{*} \gamma \wedge K-K \wedge^{*} \gamma\right) \psi \\
& \left.=L\left(\gamma, \psi, D^{\{\}} \psi\right)-\frac{1}{48}\left[\operatorname{Tr}(\check{\gamma}\rfloor^{*} \Theta\right)\right] \wedge \bar{\psi} \sigma \wedge \gamma \psi \\
& =L\left(\gamma, \psi, D^{\{\}} \psi\right)-\frac{1}{4} A \wedge \bar{\psi} \gamma_{5}{ }^{*} \gamma \psi
\end{aligned}
$$




\section{CLASSICAL AXIAL ANOMALY}

Since $L_{\mathrm{D}}=\bar{L}_{\mathrm{D}}=L_{\mathrm{D}}^{\dagger}$ is Hermitian as required, it provides us with the following charge and axial current, respectively,

$$
j=\bar{\psi}^{*} \gamma \psi, \quad j_{5}:=\bar{\psi} \gamma_{5}{ }^{*} \gamma \psi=\frac{1}{3} \bar{\psi} \sigma \wedge \gamma \psi
$$

From the Dirac equation (3.2) and its adjoint one can readily deduce the well-known "classical axial anomaly" 20]

$$
d j_{5}=2 i m P=2 i m \bar{\psi} \gamma_{5} \psi
$$

for massive Dirac fields. This also holds in a Riemann-Cartan spacetime. If we restore chiral symmetry in the limit $m \rightarrow 0$, this leads to classical conservation law of the axial current for massless Weyl spinors, or since $d j=0$, equivalently, for the chiral currrent

$$
j_{ \pm}:=\frac{1}{2} \bar{\psi}\left(1 \pm \gamma_{5}\right)^{*} \gamma \psi=\bar{\psi}_{\mathrm{L}, \mathrm{R}}{ }^{*} \gamma \psi_{\mathrm{L}, \mathrm{R}}, \quad d j_{ \pm}=0
$$

\section{SQUARED DIRAC EQUATION}

The decomposed Lagrangian (3.5), i.e.

$$
L_{\mathrm{D}}=L\left(\gamma, \psi, D^{\{\}} \psi\right)-\frac{1}{4} A \wedge j_{5}
$$

leads to the following equivalent form of the Dirac equation in $\mathrm{RC}$ spacetime

$$
i^{*} \gamma \wedge\left[D^{\{\}}+\frac{i}{4} m \gamma+\frac{i}{4} A \gamma_{5}\right] \psi=0
$$

Note that for a Riemannian covariant derivative we have $D^{\{\}} \gamma=0$. Hence, in a RiemannCartan spacetime a Dirac spinor does only feel the axial torsion one-form $A$.

Thus the Hermitian Dirac operator in a RC spacetime is the zero form

$$
\not D:=i^{*}\left[{ }^{*} \gamma \wedge\left(D^{\{\}}+\frac{i}{4} m \gamma+\frac{i}{4} A \gamma_{5}\right)\right]=i \not D^{\{\}}+\frac{1}{4} \gamma_{5} \not x-m,
$$


where the usual Feynman "dagger" convention $\left.\not A:=\check{\gamma}\rfloor A=\gamma^{\alpha} e_{\alpha}\right\rfloor A=-(-1)^{s *}\left[{ }^{*} \gamma \wedge A\right]$ for one-forms is used.

For squaring this operator, we are going to use the geometric identity (3.6.13) of [15] restricted to Riemannian spacetime, i.e.

$$
\left[D_{\alpha}^{\{\}}, D_{\beta}^{\{\}}\right]=\frac{i}{4} R_{\alpha \beta \mu \nu}^{\{\}} \sigma^{\mu \nu}
$$

where $\left.D_{\alpha}^{\{\}}:=e_{\alpha}\right\rfloor D^{\{\}}$are the components of the Riemannian covariant derivative.

Thus we obtain for the squared Dirac operator:

$$
\begin{aligned}
\not \not^{2} & =-\frac{1}{2} \gamma^{\alpha} \gamma^{\beta}\left(\left\{D_{\alpha}^{\{\}}, D_{\beta}^{\{\}}\right\}+\left[D_{\alpha}^{\{\}}, D_{\beta}^{\{\}}\right]\right)-2 i m \not D^{\{\}} \\
& -\frac{i}{4} \gamma_{5}\left(\not D^{\{\}} \not A\right)-\frac{1}{2} \gamma_{5} \sigma^{\alpha \beta} A_{\alpha} D_{\beta}^{\{\}}+m^{2}-\frac{1}{2} m \gamma_{5} \not 1-\frac{1}{16} \not A \not A \\
& \cong-\square-\frac{1}{8} \sigma^{\alpha \beta} R_{\alpha \beta \mu \nu}^{\{\}} \sigma^{\mu \nu}-\frac{i}{4} \gamma_{5}\left(\not D^{\{\}} \not A\right)-\frac{1}{2} \gamma_{5} \sigma^{\alpha \beta} A_{\alpha} D_{\beta}^{\{\}}-\frac{1}{16} A_{\alpha} A^{\alpha}-m^{2}
\end{aligned}
$$

where $\square:=\partial_{\mu}\left(\sqrt{|g|} g^{\mu \nu} \partial_{\nu}\right) / \sqrt{|g|}$ is the generally covariant Riemannian d'Alembertian operator. In the last step we used the Dirac equation. Not unexpectedly, besides the familiar Riemannian curvature scalar, only the axial torsion $A=A_{\alpha} \vartheta^{\alpha}$ contributes the the squared Dirac operator for massive spinor fields.

\section{AXIAL CURRENT IN THE EINSTEIN-CARTAN-DIRAC THEORY}

The Einstein-Cartan-Dirac (ECD) theory of a gravitationally coupled spin 1/2 fermion field provides a dynamical understanding of the axial anomaly on a classical (i.e., not quantized) level. The ECD-Lagrangian reads:

$$
L=\frac{i}{2 \ell^{2}} \operatorname{Tr}\left(\Omega \wedge^{*} \sigma\right)+L_{\mathrm{D}}=\frac{1}{2 \ell^{2}} R^{\alpha \beta} \wedge \eta_{\alpha \beta}+L_{\mathrm{D}}
$$

where $\eta^{\alpha \beta}:={ }^{*}\left(\vartheta^{\alpha} \wedge \vartheta^{\beta}\right)$ is dual to the unit two-form.

The spin current of the Dirac field is given by the Hermitian three-form

$$
\tau_{\alpha \beta}:=\frac{\partial L_{\mathrm{D}}}{\partial \Gamma^{\alpha \beta}}=\frac{1}{8} \bar{\Psi}\left({ }^{*} \gamma \sigma_{\alpha \beta}+\sigma_{\alpha \beta}{ }^{*} \gamma\right) \Psi=-\frac{1}{4} \eta_{\alpha \beta \gamma \delta} \bar{\Psi} \gamma_{5} \gamma^{\delta} \Psi \eta^{\gamma}
$$


which implies that the components $\tau_{\alpha \beta \gamma}=\tau_{[\alpha \beta \gamma]}$ of the spin current are totally antisymmetric.

The second field equation of EC-theory, i.e. Cartan's algebraic relation between torsion and spin,

$$
-\frac{1}{2} \eta_{\alpha \beta \gamma} \wedge T^{\gamma}=\ell^{2} \tau_{\alpha \beta}
$$

implies the following relation (cf. [32]) between the axial current $j_{5}$ of the Dirac field and the translational Chern-Simons term (2.5), or, equivalent, for the axial torsion one-form:

$$
C_{\mathrm{TT}} \cong \frac{1}{4} j_{5}, \quad A=2 \ell^{2 *} C_{\mathrm{TT}}=\left(\ell^{2} / 2\right) \bar{\psi} \gamma_{5} \gamma \psi
$$

Thus we find in ECD-theory

$$
d j_{5} \cong 4 d C_{\mathrm{TT}}=\frac{2}{\ell^{2}}\left(T^{\alpha} \wedge T_{\alpha}+R_{\alpha \beta} \wedge \vartheta^{\alpha} \wedge \vartheta^{\beta}\right)
$$

which establishes a link to the Nieh-Yan four form [33], but only for massive fields as will be shown below. If a coupling to the Weyl covector $Q$ is allowed for, the term $-Q \wedge j_{5}$ occurs on the right-hand side.

This result, cf. [31, 32, holds on the level of first quantization. Since the Hamiltonian of the semi-classical Dirac field is not bounded from below, one has to go over to second quantization, where the divergence of the axial current picks up anomalous terms. The question is whether in the vacuum expectation value $<d j_{5}>$ similar torsion and Weyl covector terms emerge, besides the usual Pontrjagin term.

However, if we restore chiral invariance for the Dirac fields in the limit $m \rightarrow 0$, we find within the dynamical framework of ECD theory that the Nieh-Yan four-form tends to zero "on shell", i.e.

$$
d C_{\mathrm{TT}} \cong(1 / 4) d j_{5} \rightarrow 0
$$

This is consistent with the fact that a Weyl spinor does not couple to torsion at all, because the remaining axial torsion $A$ becomes a lightlike covector, i.e. $A_{\alpha} A^{\alpha} \eta=A \wedge^{*} A \cong\left(\ell^{4} / 4\right)^{*} j_{5} \wedge$ $j_{5}=0$. Here we implicitly assume that the light-cone structure of the axial covector ${ }^{*} j_{5}$ is not spoiled by quantum corrections, i.e. that no "Lorentz anomaly" occurs as in $n=4 k+2$ dimensions 25]. 


\section{CHIRAL ANOMALY IN QUANTUM FIELD THEORY}

When quantum field theory (QFT) is involved, other boundary terms may arise in the chiral anomaly due to the non-conservation of the axial current, cf. [43, 16, 17]. An anomaly in QFT is a (classical) symmetry which is broken by field quantization. Such quantum violations were calculated for the chiral current in a torsion-free Riemannian background before [22].

Now, to approach the anomaly in the context of space-time with torsion, we will proceed by switching off the curvature and concentrate on the last term in the decomposed Dirac Lagrangian (5.1).

Then, this term can be regarded as an external axial covector $A$ (without Lorentz or "internal" indices) coupled to the axial current $j_{5}$ of the Dirac field in an initially flat spacetime. By applying the result (11-225) of Itzykson and Zuber [18, we find that only the term $d A \wedge d A$ arises in the axial anomaly, but not the Nieh-Yan type term $d^{*} A \sim d C_{\mathrm{TT}}$ as was recently claimed [6]. After switching on the curved spacetime of Riemannian geometry, we finally obtain for the axial anomaly

$$
<d j_{5}>=2 i m<P>+\frac{1}{24 \pi^{2}}\left[\operatorname{Tr}\left(R^{\{\}} \wedge R^{\{\}}\right)-\frac{1}{4} d A \wedge d A\right] .
$$

This result is based on diagrammatic techniques and the Pauli-Villars regularization scheme.

In this respect, it is a typical perturbative result. This becomes obvious if we compare it with other perturbative results: another option were to use a point-splitted current,

$$
j_{5}(x ; \epsilon):=\bar{\psi}(x) \gamma_{5}{ }^{*} \gamma \psi(x+\epsilon)
$$

where $\epsilon$ is an infinitesimal four-vector in space-time. Such an expression can be rendered invariant by dressing it with a path-ordered exponential

$$
\bar{\psi}(x) \gamma_{5}{ }^{*} \gamma \psi(x+\epsilon) \rightarrow \bar{\psi}(x) \gamma_{5}^{*} \gamma \psi(x+\epsilon) \mathbf{P} \exp \left\{i \int_{x}^{x+\epsilon} A\right\}
$$

The variation $\delta / \delta A$ of the current $j_{5}(x ; \epsilon)$ is compensated by the variation of the exponential. As the parallel transport from $x^{\mu} \rightarrow x^{\mu}+\epsilon^{\mu}$ along the infinitesimal line element can be 
expanded perturbatively, it is clear that the net effect of this approach is just the standard result $\left\langle d j_{5}(x)>=2 i m<P>-\left(1 / 96 \pi^{2}\right) d A \wedge d A\right.$ (curvature still switched off).

A further option is to use dimensional regularization. If one adopts the $\gamma_{5}$ scheme of Ref. [23], one can indeed immediately conclude that only the result (7.1) can appear. In this scheme, the only effect of the $\gamma_{5}$ problem is the replacement of the usual trace by a non-cyclic linear functional. The anomaly appears as the sole effect of this non-cyclicity and, vice versa, all non-cyclic effects are related to the anomaly as it is manifested in the triangle graphs. There is no room for other sources of non-cyclicity apart from the very fermion loops which produce the result (7.1). The whole effect of non-cyclicity is to have an operator $\Delta$, which measures the amount of violation of gauge invariance in this scheme [23]. One has $\Delta^{2}=0$, and the anomaly is in the image modulo the kernel of $\Delta$, which summarizes the fact that in this $\gamma_{5}$ scheme no other anomalous contributions are possible beside (7.1).

So, in agreement with [5, 11,40,41] we find no Nieh-Yan term in the anomaly. But at this stage we have not discussed the possibility that the torsion is not adiabatically connected to the torsion-free case. Chandia and Zanelli argue that in such a case they find a term $\sim d C_{\mathrm{TT}}$. But in their result, this term is multiplied by a factor $M^{2}$. This factor $M^{2}$ corresponds to a regulator mass in a Fujikawa type approach. This is in agreement with previous calculations, which obtained such ill-defined contributions to the anomaly as well, in other regulator methods. They were consistently absorbed in a counterterm, and thus discarded from the final result for the anomaly.

In contrast, Chandia and Zanelli argue that such contributions can be maintained by absorbing the divergent factor in a rescaled vierbein, and propose to consider the limit $\vartheta^{\alpha} \rightarrow \vartheta^{\alpha} / M$, with $M \ell$ fixed. Here, $\ell$ is the length scale introduced in the topological invariant $d C_{\mathrm{TT}}$. It is part of the definition of the topological invariant. But then, the argument of Chandia and Zanelli is highly problematic. There are at least three points which seem unsatisfactory in it.

1. First, consider $d C_{\mathrm{TT}}$. It is, by construction, a topological invariant (it is the difference 
of two Pontrjagin classes, after all, cf. Eq. (2.7). Now, it is actually not the term $d C_{\mathrm{TT}}$ which appears as the torsion-dependent extra contribution to the anomaly, but the term $2\left(T^{\alpha} \wedge T_{\alpha}+R_{\alpha \beta} \wedge \vartheta^{\alpha} \wedge \vartheta^{\beta}\right)=\ell^{2} d C_{\mathrm{TT}}$. Thus, measuring its proportion in units of the topological invariant $d C_{\mathrm{TT}}$, we find that it vanishes when we consider the proposed limit $M \rightarrow \infty, M \ell \sim$ constant.

2. The second point is that we do not have to rescale the vierbein, it is consistent to compensate the ill-defined term by a counterterm. This implies that consistently a renormalization condition can be imposed which guarantees that the anomaly has the value (7.1). Even if Chandia and Zanelli render their extra term finite by a rescaling, they have to confront the fact that a (finite) renormalization condition can be imposed which settles the anomaly at this value.

3. Finally, it is well-known that usually the appearance of a chiral anomaly is deeply connected with the presence of a conformal anomaly [34,9,21]. This makes sense: usually, conformal invariance is lost due to the (dynamical) generation of a scale. But this is the very mechanism which destroys chiral invariance as well. Thus, one would expect the Chandia and Zanelli argument to fail, as it tries to combine strict conformal invariance with a chiral anomaly.

Summarizing, our conclusions deviate sharply from the interpretation Chandi and Zanelli propose.

In the limit $m \rightarrow 0$, we obtain for the chiral anomaly Eq. (7.1). Depending on the asymptotic helicity states, there occur contributions of topological origin of the Riemannian Pointrjagin or Euler term, respectively. Interesting enough, there is a Pointrjagin type contribution $d A \wedge d A$ from axial torsion in Riemann-Cartan spacetime. Its role for the topology of contorted spacetimes is rather unexplored, cf. [13,8].

In the next two sections we will further strengthen our argument. We will consider a situation where a cosmic string with a torsion line defect is present. This is a situation where torsion is indeed realized in a discontinous manner. Nevertheless, we will see that the 
Nieh-Yan term vanishes identically. Also, we will discuss the heat kernel method in some detail, pointing out the various scaling properties which are related with the fact that $K_{2}$ has the wrong dimensionality in four dimensions.

\section{SPINNING COSMIC STRING WITH TORSION}

In order to analyze also spacetimes with torsion defects or singularities, let us consider a cosmic string solution within the Einstein-Cartan (EC) theory governed by the field equations

$$
-\frac{1}{2} \eta_{\alpha \beta \gamma} \wedge R^{\beta \gamma}=\ell^{2} \Sigma_{\alpha}
$$

and the Cartan relation (6.3).

Let us adopt here the convention that $x^{\alpha}$ together with $y^{\alpha}$ are spacelike orthogonal vectors which span the $(x, y)$-plane orthogonal to the $(t, z)$-plane, the world sheet of the string. The corresponding one-forms are denoted by capital letters, i.e.

$$
X:=x_{\alpha} \vartheta^{\alpha}, \quad Y:=y_{\alpha} \vartheta^{\alpha} .
$$

Moreover, the vector $n^{\alpha}$ is a timelike unit vector normal to the hypersurface with $n^{\alpha} n_{\alpha}=s$, the signature $s$ of spacetime.

Following Soleng [36], cf. Anandan [1], we assume that three-forms $\Sigma_{\alpha}$ and $\tau_{\alpha \beta}$ of the energy-momentum and spin current, repectively, vanish outside of the string, whereas "inside" they are constant, i.e.

$$
\Sigma_{\alpha}=\varepsilon \vartheta_{\alpha} \wedge X \wedge Y, \quad \tau_{\alpha \beta}=\sigma \eta_{\alpha \beta \gamma} n^{\gamma} \wedge X \wedge Y
$$

The constant parameters $\varepsilon$ and $\sigma$ of this spinning string are related to the exterior vacuum solution by appropriate matching conditions. For the related solution with conical singularities and torsion of Tod [39], $\varepsilon$ and $\sigma$ are delta distributions at the location of the string. From the specification (8.2) of the one-forms $X$ and $Y$ it can easily be infered that the only nonzero components are $\Sigma_{\hat{0}} \neq 0, \Sigma_{\hat{3}} \neq 0$ and $\tau_{\hat{1} \hat{2}}=-\tau_{\hat{2} \hat{1}} \neq 0$. 
Since $x^{\alpha} \Sigma_{\alpha}=y^{\alpha} \Sigma_{\alpha}=0$, contractions of the first EC field equation (8.1) with $x^{\alpha}$ and $y^{\alpha}$ reveal that $x^{[\alpha} y^{\beta]} R_{\alpha \beta}=R_{\hat{1} \hat{2}}=-R_{\hat{2} \hat{1}} \neq 0$ are the only nonvanishing components of the $\mathrm{RC}$ curvature. From its covariant expression [26]

$$
R^{\alpha \beta}=\varepsilon \ell^{2} x^{[\alpha} y^{\beta]} X \wedge Y
$$

there follows the identity

$$
R_{\beta}{ }^{\alpha} \wedge \vartheta^{\beta}=\frac{\varepsilon \ell^{2}}{2}\left(x^{\alpha} Y \wedge X \wedge Y-y^{\alpha} X \wedge X \wedge Y\right)=0 .
$$

From the Cartan relation (6.3) we find for the torsion

$$
T^{\alpha}=-2 \sigma \ell^{2} n^{\alpha} X \wedge Y \quad \Rightarrow \quad T_{\alpha} \wedge T^{\alpha}=4 s \sigma^{2} \ell^{4} X \wedge Y \wedge X \wedge Y=0 .
$$

Recalling that $\left.N^{\alpha}=n\right\rfloor \vartheta^{\alpha}$ is the lapse and shift vector in the (3+1)-decomposition of the ADM formalism, the corresponding coframe and connection can now explicitly be obtained after applying a finite boost to the usual conical metric of the cosmic string, cf. [1]

$$
\begin{aligned}
\vartheta^{\hat{0}} & =d t+\ell^{2} \sigma \rho^{* 2}\left[1-\cos \left(\rho / \rho^{*}\right)\right] d \phi \\
\vartheta^{\hat{1}} & =d \rho, \quad \vartheta^{\hat{2}}=\rho^{*} \sin \left(\rho / \rho^{*}\right) d \phi, \quad \vartheta^{\hat{3}}=d z, \\
\Gamma^{\hat{1} \hat{2}} & =\cos \left(\rho / \rho^{*}\right) d \phi=-\Gamma^{\hat{2} \hat{1}} .
\end{aligned}
$$

Thus from the identities (8.6) and (8.5), we can infer that the Nieh-Yan term (2.6) vanishes identically for this example of a spinning cosmic string exhibiting a torsion line defect. The same holds for the stationary cosmic string solution of Letelier [24], see also Ref. [2], where the vector normal to the hypersurface is generalized to $n^{\alpha}=(1,0,0, \tau / \sigma)$.

\section{COMPARISON WITH THE HEAT KERNEL METHOD}

In the heat kernel approach, there exists for small $t \rightarrow+0$ an asymptotic expansion of the kernel in $n$ dimensions:

$$
K\left(t, x, x, \not \not^{2}\right)=(4 \pi)^{-n / 2} \sum_{k=0}^{\infty} t^{(k-n) / 2} K_{k}\left(x, \not \not^{2}\right)
$$


The coefficients $K_{k}\left(x, \mathcal{D}^{2}\right), k=0,1, \ldots$ are completely determined by the form of the second-order differential operator $\not \not^{2}$, which is positive for Euclidean signature diag $o_{\alpha \beta}=$ $(-1, \cdots,-1)$. For odd $k=1,3, \ldots$ these coefficients are zero, while the first nontrivial terms 35, 38,40, which potentially could contribute to the axial anomaly, read

$$
\begin{aligned}
& \operatorname{Tr}\left(\gamma_{5} K_{2}\right)=-d^{*} A \\
& \operatorname{Tr}\left(\gamma_{5} K_{4}\right)=\frac{1}{6}\left[\operatorname{Tr}\left(R^{\{\}} \wedge R^{\{\}}\right)-\frac{1}{4} d A \wedge d A+d \mathcal{K}\right] .
\end{aligned}
$$

However, there is an essential difference in the physical dimensionality of the terms $K_{2}$ and $K_{4}$. Whereas in $n=4$ dimensions the Pontrjagin type term $K_{4}$ is dimensionless and thus multiplied by $t^{(k-4) / 2}=1$, the term $K_{2} \sim d^{*} A=2 \ell^{2} d C_{\mathrm{TT}}$ carries dimensions. Since a massive Dirac spinor has canonical dimension $[\text { length }]^{-3 / 2}$, it scales as $\psi \sim m^{3 / 2}$. Moreover, in Fujikawa method, cf. [20], the term $t=1 / M^{2}$ is related to the regulator mass $M \rightarrow \infty$. Then the second order term in the heat kernel expansion scales as

$$
-\frac{1}{t} K_{2}=\frac{2 \ell^{2}}{t} d C_{\mathrm{TT}} \cong \frac{\ell^{2}}{2 t} d j_{5}=\frac{i m \ell^{2}}{t} \bar{\psi} \gamma_{5} \psi \sim \ell^{2} M^{2} m^{4} \rightarrow 0
$$

If we assume in the renormalization procedure, that the fundamental length $\ell$ does not scale (no running coupling constant), the second order term in the heat kernel expansion will tend to zero in the limit $m \rightarrow 0$. In the case $m \neq 0$, this term diverges and the Fujikawa regulator method cannot be applied. To rescale the coframe by $\vartheta^{\alpha} \rightarrow \vartheta^{\alpha} / \ell$ does not help, since this would change also the dimension of the Dirac field, in order to retain the physical dimension $[\hbar]$ of the Dirac action.

Then our conclusion is that that Nieh-Yan term $d C_{\mathrm{TT}}$ does NOT contribute to the chiral anomaly in $n=4$ dimensions, neither classically nor in quantum field theory, as pointed out before. This is in sharp contrast to the recent paper of Chandia [6] et al. We once more stress the interrelation between the scale and chiral invariance [34,9,21]. Since renormalization amounts to a continous scale deformation, only the Riemannian part of the Pontrjagin term contributes to the topology of the chiral anomaly, cf. [42,27]. On would surmize that in $n=2$ dimensional models only the term $d^{*} A$ survives in the heat kernel 
expansion, since it then has the correct dimensions. However, it is well-known [15] that in 2D the axial torsion $A$ vanishes identically.

\section{DISCUSSION}

We have shown that the translational Chern-Simons boundary term $d C_{\mathrm{TT}}$, which in the canonical formulation á la Ashtekar plays the role of a generating functional [29] for chiral (self- or antiself-dual) variables in Einstein-Cartan-Dirac theory as well as in simple supergravity [31], is not affected by the chiral anomaly. The appearance of the Riemannian Pontrjagin term $d C_{\mathrm{RR}}$ could pose some problems for the canonical approach of gravity á la Ashtekar. This is likely the case, although the state

$$
\Psi_{\Lambda}=\exp \left(\frac{3}{\Lambda} \int_{M_{3}} \underline{C}_{\mathrm{RR}}\right)
$$

involving the tangential complexified Chern-Simons term $\underline{C}_{\mathrm{RR}}$ is known to solve the Hamiltonian constraint of gravity with cosmological constant $\Lambda$ in the loop approach [4, 12]. More-

over, the additional Pointrjagin type term $d(A \wedge d A)$ arising from the axial torsion $A$, necessarily present in the case gravitationaly coupled Dirac and Rarita-Schwinger fields, could turn out to be a major obstacle for completing the canonical approach of gravity a la Ashtekar, on top of the open issue of reality conditions, cf. [37].

\section{ACKNOWLEDGMENTS}

We would like to thank Alfredo Macías, Hugo Maroles-Técotl, and Yuri Obukhov for useful hints and comments on a preliminary version. This work was partially supported by CONACyT, grant No. 3544-E9311, and by the joint German-Mexican project KFAConacyt E130-2924. One of us (E.W.M.) acknowledges the support by the short-term fellowship 9616160156 of the German Academic Exchange Service (DAAD), Bonn. D.K. acknowledges support by a Heisenberg fellowship of the DFG. 


\section{REFERENCES}

[1] J. Anandan, Phys. Lett. A195 (1994) 284.

[2] J. Anandan, Phys. Rev. D53 (1996) 779.

[3] M. Atiyah: "The Dirac equation and geometry" (Trinity College preprint 1996).

[4] B. Brügmann, R. Gambini, and J. Pullin, Nucl. Phys. B385 (1992) 587.

[5] I.L. Buchbinder, S.D. Odintsov, and I.L. Shapiro: Effective Action in Quantum Gravity. IOP Publishing (Bristol, 1992); see, in particular, Chapter 4.

[6] O. Chandia and J. Zanelli: Topological invariants, instantons and chiral anomaly on spaces with torsion, Preprint Santiago de Chile, hep-th/9702025.

[7] G. Cognola and S. Zerbini, Phys. Lett. B214, 70 (1988).

[8] A.D. Dolgov, I.B. Khriplovich, and V.I. Zakharov, Nucl. Phys. B309, 591 (1988).

[9] J. Ellis, Nucl. Phys. B22 (1970) 478.

[10] S. Gotzes and A.C. Hirshfeld, Ann. Phys. (N.Y.) 203 (1990) 410.

[11] G. Grensing, Phys. Lett. B169 (1986) 333.

[12] J. Griego, Phys. Rev. D53 (1996) 6966.

[13] A.J. Hanson and T. Regge, in: Lecture Notes in Physics, Vol. 94 (Springer, Berlin 1979 ), p. 354 .

[14] F.W. Hehl, W. Kopczyński, J.D. McCrea, and E.W. Mielke, J. Math. Phys. 32 (1991) 2169-2180.

[15] F.W. Hehl, J.D. McCrea, E.W. Mielke, and Y. Ne'eman, Phys. Rep. 258 (1995) 1-171.

[16] A.C. Hirshfeld, in: Proc. of the School on Geometry and Theoretical Physics, Bad Honnef, 12. -16. Feb. 1990, J. Debrus and A.C. Hirshfeld, eds. (Springer, Berlin 1991), 
p. 178.

[17] B.R. Holstein, Am. J. Phys. 61 (1993) 142.

[18] C. Itzykson and J.-B. Zuber: Quantum Field Theory (McGraw Hill, New York 1980).

[19] J. Julve, A. López-Pinto, A. Tiemblo, and R. Tresguerres, Gen. Rel. Grav. 28 (1996) 759.

[20] M. Kaku: Quantum Field Theory (Oxford University Press 1993). p. 424.

[21] G. Kälbermann, Phys. Rev. D 42 (1990) 2893.

[22] T. Kimura, Prog. Theo. Phys. 42 (1969) 1191;

R. Delbourgo and Abdus Salam, Phys. Lett. B40 (1972) 381;

T. Eguchi and P. Freund, Phys. Rev. Lett. (1976) 1251.

[23] D. Kreimer, Phys. Lett. B237 (1990) 59;

J.G. Körner, D. Kreimer, K. Schilcher, Z.Phys.C54 (1992) 503;

"The role of $\gamma_{5}$ in dimensional regularization", UTAS-PHYS-94-01 (revised version in preparation), hep-ph/9401354.

[24] P.S. Letelier, Class. Quantum Grav. 12 (1995) 471.

[25] H. Leutwyler, Helvetia Physica Acta 59 (1986) 201.

[26] A. Macías, A., E.W. Mielke, and H.A. Morales-Técotl, in: New Frontiers in Gravitation, G.A. Sardanashvily, ed. (Hadronic Press, Palm Harbor FL, 1996), pp. 227 - 242.

[27] E.W. Mielke, Phys. Lett. A110 (1985) 87-91.

[28] E.W. Mielke: Geometrodynamics of Gauge Fields — On the geometry of Yang-Mills and gravitational gauge theories (Akademie-Verlag, Berlin 1987).

[29] E.W. Mielke, Ann. Phys. (N.Y.) 219 (1992) 78-108.

[30] E.W. Mielke, J.D. McCrea, Y. Ne'eman, and F.W. Hehl, Phys. Rev. D48 (1993) 673. 
[31] E.W. Mielke, P. Baekler, F.W. Hehl, A. Macías, and H.A. Morales-Técotl, in: Gravity, Particles and Space-Time, ed. by P. Pronin and G. Sardanashvily (World Scientific, Singapore, 1996), pp. 217-254.

[32] E.W. Mielke, A. Macías, and H.A. Morales-Técotl, Phys. Lett. A 215 (1996) 14-20.

[33] H.T. Nieh and M.L. Yan, J. Math. Phys. 23 (1982) 373-374.

[34] Abdus Salam and J. Strathdee, Phys. Rev. 184 (1969) 1760;

G. Mack, and Abdus Salam, Ann. Phys. 53 (1969) 174;

C.J. Isham, Abdus Salam, and J. Strathdee, Phys. Lett. B31 (1970) 300.

[35] Yu.N. Obukhov, Nucl. Phys. B212 (1983) 237; Phys. Lett. B108 (1982) 308-310.

[36] H.H. Soleng, Gen. Rel. Grav. 24 (1992) 111.

[37] T. Thiemann, Phys. Lett. B380 (1996) 257.

[38] Yu. N. Obukhov, E.W. Mielke, J. Budczies, and F.W. Hehl: "On the chiral anomaly in non-Riemannian spacetimes." gr-qc/9702011.

[39] K.P. Tod, Class. Quantum Grav. 11 (1994) 1331.

[40] S. Yajima, Class. Quantum Grav. 13 (1996) 2423-2435.

[41] C. Wiesendanger, Class. Quant. Grav. 13 (1996) 681-699.

[42] Y. S. Wu and A. Zee, J. Math. Phys. 25, 2696 (1984).

[43] B. Zumino, in B.S. DeWitt and R. Stora, eds. Relativity, Groups and Topology II, (Elsevier Science, Publ. 1984), pp. $1292-1322$. 